\title{
Neuroretinitis and Juxtapapillary Retinochoroiditis as Atypical Presentations of Ocular Toxoplasmosis
}

\author{
Nelly N Kabedi \\ Jean-Claude Mwanza ${ }^{1,2}$ \\ 'Department of Ophthalmology, School \\ of Medicine, University of Kinshasa, \\ Kinshasa, Democratic Republic of the \\ Congo; '2Department of Ophthalmology, \\ School of Medicine, University of North \\ Carolina at Chapel Hill, Chapel Hill, \\ NC, USA
}

\begin{abstract}
We report three cases of optic nerve toxoplasmosis, an unusual form of ocular toxoplasmosis. In one patient, the optic nerve involvement occurred in an eye with a toxoplasmic chorioretinal scar and choroidal neovascularization in the supramacular area, subretinal fibrosis, and pigment epithelium detachment. The other two patients had papilledema without healed or active chorioretinal lesions, but both had retinal hemorrhage and macular involvement. The diagnosis was based on clinical examination and elevated serum toxoplasma antibodies. Optical coherence tomography helped uncover the structural chorioretinal changes. All patients were treated with a combination of oral antitoxoplasmic drugs, oral prednisone, and intravitreal injection of bevacizumab. Visual acuity improved in all of them. Optic nerve involvement in ocular toxoplasmosis must be considered when papilledema occurs both in isolation and/or in the presence of an active or scarred chorioretinal lesion.
\end{abstract}

Keywords: optic nerve toxoplasmosis, neuroretinitis, neovascularization, retinal hemorrhage

\section{Introduction}

Toxoplasmosis can affect several organs, including the eye. Ocular toxoplasmosis (OT) may result from either congenital or acquired infection after birth. ${ }^{1}$ Approximately $20 \%$ of infected people develop OT, which is the leading cause of posterior infectious uveitis in immunocompetent people. ${ }^{1,2}$ OT in the form of retinochoroiditis can account for up to $85 \%$ of all infectious causes of posterior uveitis. ${ }^{2}$ Its hallmark sign is a focal necrotizing retinitis associated or not with vitritis and anterior uveitis. Regardless of whether it is treated or not, retinal necrosis heals by leaving a pigmented retinochoroidal scar. Toxoplasma-induced retinal choroiditis can also manifest itself as multiple gray-white punctate lesions deep in the retina. ${ }^{3,4}$ Optic nerve toxoplasmosis is a less common presentation and is believed to occur in $5.3 \%$ to $16.5 \%$ of all OT. ${ }^{5}$ Involvement of the optic nerve typically occurs next to or far from an active retinochoroiditis lesion. It can also present without an active or scarred lesion as papillitis or neuroretinitis. ${ }^{5}$ Sometimes, it could be accompanied by vitritis and/or anterior uveitis, but some other times there is neither vitritis nor anterior uveitis. ${ }^{6}$ Posterior segment complications of OT include, among others, cystoid macular edema, retinal detachment, retinal artery or vein occlusion, vasculitis, and subretinal and choroidal neovascularization $(\mathrm{CNV}) .^{7-9} \mathrm{CNV}$ is a rare and late complication of toxoplasmic chorioretinitis. We report three cases of optic nerve toxoplasmosis, which were presented with juxtapapillary retinochoroiditis and papillitis with some complications.
Correspondence: Nelly N Kabedi Department of Ophthalmology, School of Medicine, University of Kinshasa, Kinshasa, Democratic Republic of the Congo

Tel +243-97I-183-249

Email nelnkabedi@gmail.com 


\section{Case Description}

\section{Case \#I}

A 56-year-old woman with unremarkable past medical and ophthalmological history presented with blurry vision in the right eye that started two months earlier. Visual acuity was limited to counting fingers in the right and 1.0 in the left eye. The anterior segment was free of inflammatory signs in both eyes. There were no signs of vitritis either. Intraocular pressure (IOP) was $16 \mathrm{mmHg}$ in both eyes. Fundus examination revealed papilledema covering the upper half of the optic disc, a chorioretinal scar, and fibrotic lesion near the macula in the right eye (Figure 1). The left eye appeared normal. Optical coherence tomography (OCT) of the right eye showed an elevated optic disc, thickening of peripapillary retinal fiber layer (RNFL) in the superior, inferior and nasal sectors, pigment epithelium detachment (PED), small pockets of intraretinal fluid, and subretinal fibrosis in the macula area (Figure 1). OCT examination of the left eye was normal. The average central retinal thickness was $361 \mu \mathrm{m}$ in the right and $193 \mu \mathrm{m}$ in left eye. The average peripapillary RNFL thickness was $172 \mu \mathrm{m}$ and $121 \mu \mathrm{m}$ in the right and left eye, respectively. Serum toxoplasmosis antibody titers were $77 \mathrm{IU} / \mathrm{mL}$ for IgM and $0.01 \mathrm{IU} / \mathrm{mL}$ for IgG. The diagnosis of toxoplasma-related juxtapapillary retinochoroiditis was made, with pre-existing CNV from the old episode. The patient was treated with a combination of oral antiparasitic drugs (association pyrimethamine $50 \mathrm{mg}$ / day, sulfadiazine $2 \mathrm{~g}$ on the first day and then $1 \mathrm{gr} /$ day thereafter, rovamycin $2 \times 3 \mathrm{IU} /$ day), folinic acid $10 \mathrm{mg}$ /day, oral prednisone $1 \mathrm{mg} / \mathrm{kg}$ on the first day and then $0.5 \mathrm{mg} /$ day thereafter, and intravitreal injection of $0.05 \mathrm{~mL}$ $(1.25 \mathrm{mg})$ of bevacizumab (Avastin, Roche, Basel, Switzerland). She received a total of 3 intravitreal injections, one per month and parasitic drugs for 4 weeks. A month later, visual acuity in the right eye increased to 0.5 , fundus photography showed no change relative to baseline, and OCT scan revealed persistence of the optic disc elevation (Figure 1) and complete resorption of the intraretinal fluid (Figure 1). The patient was advised to pursue with another round of antiparasitic drugs.

\section{Case \#2}

A 59-year-old female patient presented with intermittent redness in both eyes, more pronounced in the left eye for the last 2 months. She also had pain, blurred vision and myodesopsia in her left eye. Similar episodes during the last two years and rheumatism were recorded. Best corrected visual acuity was $9 / 10$ in the right and 5/10 in the left eye. Anterior segment and fundus examinations of the right eye were unremarkable. The left eye showed keratic precipitates, $1+$ anterior chamber cells and 2+ anterior vitreous cells. Fundus examination of the left eye showed papilledema with exudates and hemorrhage in the papillomacular bundle. Peripapillary and macular OCT scans were normal in the right eye but showed an epiretinal membrane, macular edema, and intraretinal exudates in the left eye. Average peripapillary RNFL thickness was greater in the left $(234$ $\mu \mathrm{m})$ than the right eye $(138 \mu \mathrm{m})$, with the increase in thickness affecting more the superior and nasal quadrants. Toxoplasmosis antibody titers were $192 \mathrm{IU} / \mathrm{mL}$ for $\mathrm{IgG}$ and $0.04 \mathrm{IU} / \mathrm{mL}$ for IgM. She was diagnosed with toxoplasma
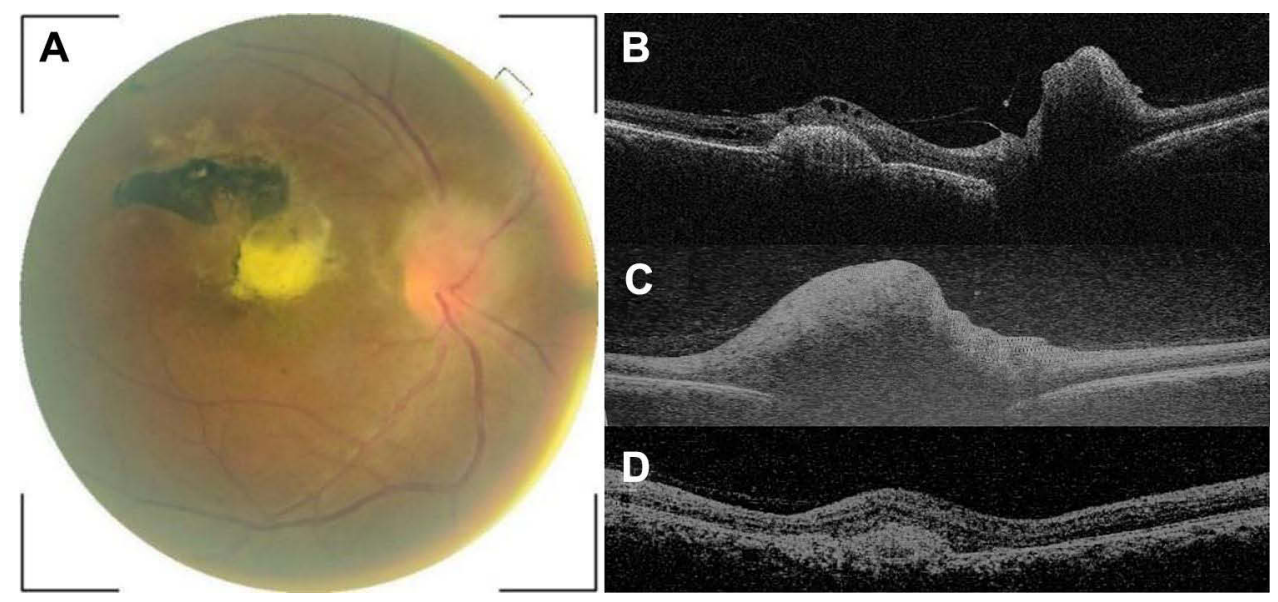

Figure I Baseline right eye fundus fundus photo of patient \#I showing papilledema in the superior part of optic disc and chorioretinal scar and fibrosis near the macula (A). Baseline OCT (B) shows optic disc elevation, pigment epithelium detachment with a few pockets of intraretinal liquid and subretinal fibrotic tissue in the macula area. After treatment, there was no apparent change on fundus photograph whereas OCT scans shows persistence of the disc elevation (C) and resorption of the intraretinal liquid (D). 
neuroretinitis and started with oral antitoxoplasmosis drugs (sulfadiazine, pyrimethamine, and rovamycin) as described for patient \#1, oral folinic acid, and oral, as well as corticosteroid eye drops. Five weeks later, visual acuity was $7 / 10$ in the left eye, and both keratitic and vitreous cells were no longer present. Papilledema decreased substantially, retinal hemorrhage completely resorbed, but exudates remained unchanged. She unfortunately suffered a kidney failure more likely due to sulfadiazine, and the parasitic drugs were discontinued.

\section{Case \#3}

A 62-year-old woman complained of pain and redness of left eye during the last 48 hours. She had a history of insomnia and peripheral spondyloarthritis for which she was under care. The best corrected visual acuity was 10/10 in both eyes. Anterior segment examination showed conjunctival hyperemia with perikeratic circle, keratic precipitates and posterior synechiae from 6 to 10 o'clock in the right and at 9 o'clock in the left eye, with diffuse iris atrophy in both eyes. IOP was $15 \mathrm{~mm} \mathrm{Hg}$ in the right and $13 \mathrm{~mm} \mathrm{Hg}$ in the left eye. Fundus examination was normal in both eyes. The patient was diagnosed with bilateral chronic anterior uveitis and treated with oral prednisone and atropine eye drops. Symptoms and signs progressively improved. One month later, she returned with tearing and mild lagophthalmos in her right eye due to right side facial palsy treated with prednisolone (unspecified dose) and unspecified vitamin pills. Visual acuity was 0.6 and 0.8 in right and left eye, respectively. Anterior and posterior segment examinations were unremarkable. IOP was $16 \mathrm{mmHg}$ in both eyes. She continued with the same treatment. One year after her first visit, she returned again, complaining of blurred vision in both eyes and reduction of visual field in the left eye. Visual acuity was 0.9 in the right and 0.5 in the left eye. Anterior segment examination showed mutton fat keratic precipitates in both eyes (more severe in the left eye). Pupils were regular with sluggish reactivity to light, there were pigments on the anterior lens capsule and signs of vitritis with $2+$ cells in the left eye. Fundus examination was normal in the right eye, whereas the left eye had papilledema with superotemporal juxtapapillary hemorrhage resembling a branch retinal vein occlusion (Figure 2). OCT scans revealed posterior vitreous detachment in both eyes with papilledema in the left eye (Figure 2) and small retrofoveal retinal detachment in the right eye. Serum toxoplasmosis antibody titers were $275 \mathrm{IU} / \mathrm{mL}$ for $\mathrm{IgG}$ and $0.2 \mathrm{IU} / \mathrm{mL}$ for

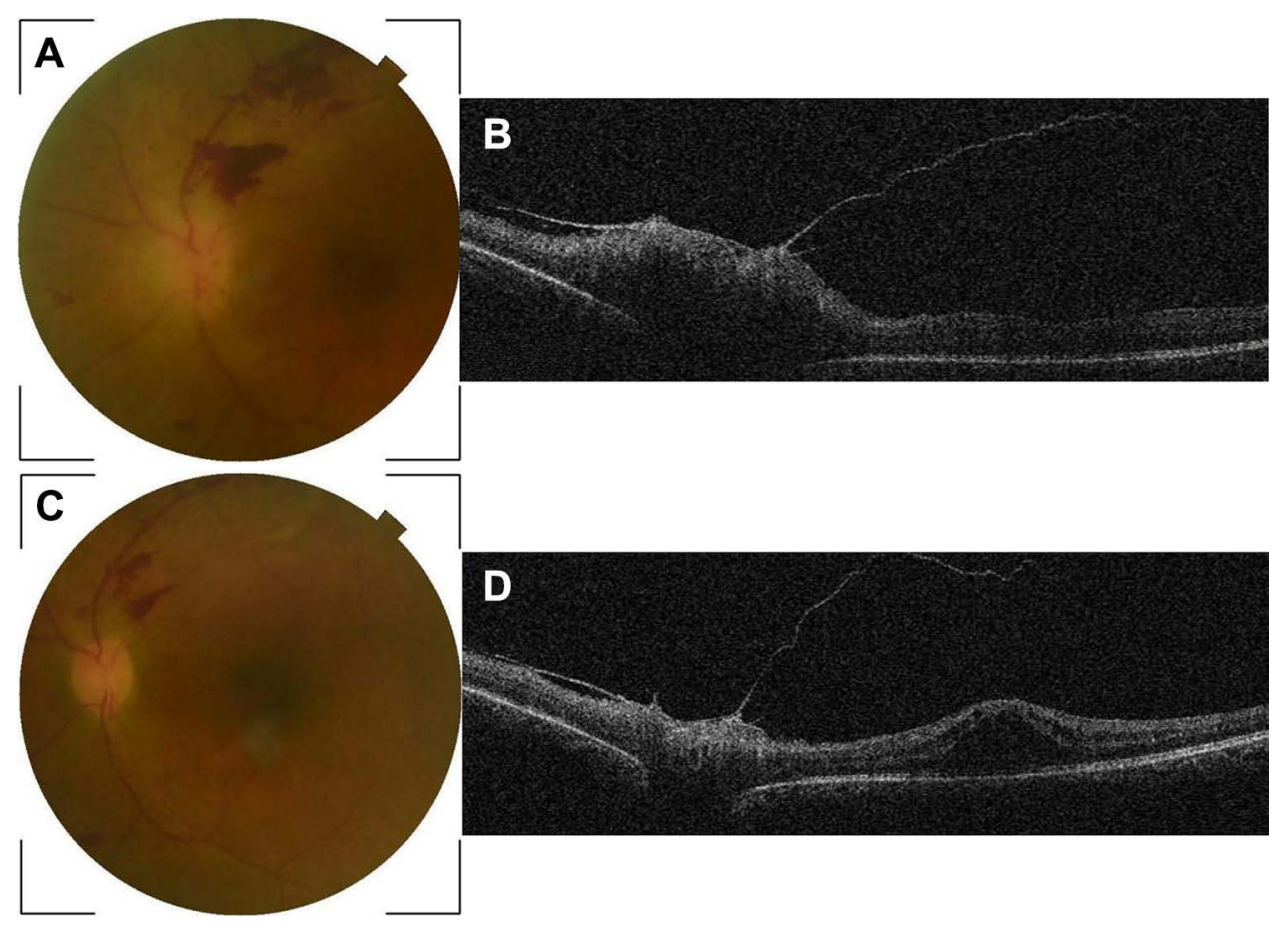

Figure 2 Baseline fundus photo $(\mathbf{A})$ of the left eye of patient \#3 displaying papilledema with retinal hemorrhage along the superotemporal retinal vein. OCT scan shows elevation of the optic disc, an epiretinal membrane, posterior vitreous detachment (PVD), and slight thickening of the macula (B). Post-treatment imaging depicts almost complete resorption of the papilledema (C), substantial resorption of the retinal hemorrhage $(\mathbf{C})$, significant reduction in optic disc elevation (D), PVD, and a macular serous detachment (D). 
IgM. She was treated for neuroretinitis with the combination of sulfadiazine, pyrimethamine, and rovamycin using the same doses as for patients \#1 and 2. She also received folinic acid $10 \mathrm{mg} /$ day and oral prednisone $1 \mathrm{mg} / \mathrm{kg}$ loading dose and $0.5 \mathrm{mg} / \mathrm{kg} /$ day subsequently. A month later, she was asymptomatic. Visual acuity improved to 1.0 in the right and 0.6 in the left eye. There were no keratic precipitates and vitreous cells. IOP was $12 \mathrm{mmHg}$ and 11 $\mathrm{mmHg}$ in right and left eye, respectively. Fundus examination was normal in the right eye and showed partial resorption of both papilledema and retinal hemorrhage in the left eye (Figure 2). OCT imaging showed retinal reattachment in the right eye and decreased papilledema and retinal hemorrhage. At the 2-month follow-up visit, she complained again of redness and blurred vision in left eye, which started after she stopped all medications due to side effects. Visual acuity decreased to 0.8 in the right eye but remained stable in the left eye. There were keratic precipitates in both eyes, but more pronounced in the left eye. Ophthalmoscopic assessment was normal in the right eye and showed substantial papilledema decrease and retinal hemorrhage in the left eye. OCT was unremarkable in the right eye. In the left eye, it confirmed the significant decrease of papilledema but revealed an important macular edema (Figure 2). Antiparasitic drugs were reinstated along with oral and topical corticosteroids.

\section{Discussion}

The clinical presentation of toxoplasmosis in our patients covers two types of optic nerve involvement, namely juxtapapillary retinochoroiditis and neuroretinitis. These types of presentation occur infrequently and the particularity of these cases resides in the other signs accompanying the optic nerve involvement. In addition to papilledema, the first patient had CNV in the same area as the chorioretinal scar. CNV complicating ocular toxoplasmosis has a prevalence between $2 \%$ and $19 \%$. CNV has been observed in association with healed toxoplasma chorioretinal lesions ${ }^{10}$ (as observed in the first patient), but it can also rarely occur during the active phase of a chorioretinitis. $^{11,12}$ Management options for $\mathrm{CNV}$ in healed toxoplasma chorioretinitis include observation, laser photocoagulation, photodynamic therapy, corticosteroids and intravitreal injection of anti-vascular endothelial growth factor (VEGF) agents. ${ }^{13}$ Because the optic nerve involvement was active and coexisted with a healed chorioretinitis with $\mathrm{CNV}$, the patient was treated with a combination of anti-VEGF and antiparasitic drugs, with favorable outcome. Functional improvement following bevacizumab intravitreal injection for CNV in toxoplasmosis has been reported in the literature. ${ }^{13}$ The use of antiparasitic drugs at the same time as anti-VEGF treatment is to prevent inflammation reactivation that can be induced by the intravitreal anti-VEGF agent. ${ }^{13,14}$ It would have been more informative to perform OCT angiography (OCTA) in our patients. Indeed, OCTA allows good characterization of vascular and hemodynamic changes in both active and healed toxoplasmic retinochoroiditis. Because the retinal vascular endothelium is highly vulnerable to T. gondii in comparison to vascular endothelium in other tissues, ${ }^{15}$ patients with toxoplasmic retinochoroiditis are at higher risk of developing ischemic or hemorrhagic events. $^{16,17}$ OCTA may therefore be used for noninvasive assessment of the perfusion of the capillary networks at different levels as well as of the choroidal vessels in the macula, retinal periphery and optic nerve. Some of the changes that have been reported include pure retinal neovascularization, choroidal neovascularization, reduced retinal microvasculature and/or choriocapillaris. ${ }^{18-20}$

The rest of the patients had neuroretinitis without chorioretinal scars in the context of chronic anterior uveitis. Both had retinal hemorrhage and one of them also had exudative retinal detachment. Optic nerve involvement in OT may present in various forms, such as juxtapapillary chorioretinitis, neuroretinitis, pure papillitis, distant to an active chorioretinitis lesion, and various combinations of these. ${ }^{21}$ When the optic nerve is affected without any chorioretinal scar or active lesion, asserting the diagnosis OT-related papillitis based on clinical findings may be difficult, but serologic tests may provide evidence of exposure to the parasite. ${ }^{21}$ Based on clinical and OCT findings, patient $\# 1$ had juxtapapillary retinochoroiditis, whereas patients \#2 and \#3 had neuroretinitis.

In all of them, visual acuity improved after treatment. The increase in visual acuity is known to differ among the different types of toxoplasma-related optic nerve disease and is small or inexistent if the macular is affected. ${ }^{21}$ This is supported by previous studies showing that visual acuity improvement is often greater in pure papillitis than in papillitis with concurrent chorioretinal lesions. ${ }^{6,21}$ However, other studies suggested that the pre-treatment visual acuity is often lower in pure papillitis than papillitis with macular involvement. ${ }^{6}$ We believe that the pre- and post-treatment visual acuity depend more on the presence of macular lesions more than the optic nerve involvement.

To conclude, clinicians should be aware of toxoplasmic optic nerve disease in the presence of papilledema. The 
diagnosis is easier clinically when papilledema is associated with an active or a scarred lesion of chorioretinitis. In isolated optic nerve involvement, serologic tests can help guide the diagnosis.

\section{Declarations}

Ethics approval and consent to participate were obtained. All procedures performed in accordance with the ethical standards of the University of Kinshasa School of Public Health and with the Declaration of Helsinki and its later amendments or comparable ethical standards. Informed consent was obtained from all participants.

\section{Informed Consent and Consent for Publication}

Patients provided informed consent to publish their case details and any accompanying images.

\section{Disclosure}

The authors declare that they have no competing interests to disclose.

\section{References}

1. Furtado JM, Smith JR, Belfort R, Gattey D, Winthrop KL. Toxoplasmosis: a global threat. J Glob Infect Dis. 2011;3:281-284. doi:10.4103/0974-777X.83536

2. Holland GN. Ocular toxoplasmosis: a global reassessment. Part I: epidemiology and course of disease. $\mathrm{Am} J$ Ophthalmol. 2003;136:973-988. doi:10.1016/j.ajo.2003.09.040

3. Bosch-Driessen LE, Berendschot TT, Ongkosuwito JV, Rothova A. Ocular toxoplasmosis: clinical features and prognosis of 154 patients. Ophthalmology. 2002;109:869-878. doi:10.1016/S0161-6420(02) 00990-9

4. Delair E, Latkany P, Noble AG, Rabiah P, McLeod R, Brezin A. Clinical manifestations of ocular toxoplasmosis. Ocul Immunol Inflamm. 2011;19:91-102. doi:10.3109/09273948.2011.564068

5. Ngoma DB, Detry-Morel M, Kayembe DL. Toxoplasma papillitis without vitritis. A case report in an immunocompetent Congolese patient. Germs. 2019;9:95-101. doi:10.18683/germs.2019.1163

6. Eckert GU, Melamed J, Menegaz B. Optic nerve changes in ocular toxoplasmosis. Eye. 2007;21:746-751. doi:10.1038/sj.eye.6702319

7. Bosch-Driessen LH, Karimi S, Stilma JS, Rothova A. Retinal detachment in ocular toxoplasmosis. Ophthalmology. 2000;107:36-40. doi:10.1016/S0161-6420(99)00013-5

International Medical Case Reports Journal

\section{Publish your work in this journal}

The International Medical Case Reports Journal is an international, peer-reviewed open-access journal publishing original case reports from all medical specialties. Previously unpublished medical posters are also accepted relating to any area of clinical or preclinical science. Submissions should not normally exceed 2,000 words or 4
8. Cotliar AM, Friedman AH. Subretinal neovascularisation in ocular toxoplasmosis. Br J Ophthalmol. 1982;66:524-529. doi:10.1136/ bjo.66.8.524

9. Gentile RC, Berinstein DM, Oppenheim R, Walsh JB. Retinal vascular occlusions complicating acute toxoplasmic retinochoroiditis. Can J Ophthalmol. 1997;32:354-358.

10. Fine SL, Owens SL, Haller JA, Knox DL, Patz A. Choroidal neovascularization as a late complication of ocular toxoplasmosis. Am J Ophthalmol. 1981;91:318-322. doi:10.1016/ 0002-9394(81)90283-X

11. Hegde S, Relhan N, Pathengay A, et al. Coexisting choroidal neovascularization and active retinochoroiditis-an uncommon presentation of ocular toxoplasmosis. $J$ Ophthalmic Inflamm Infect. 2015;5:22. doi:10.1186/s12348-015-0051-2

12. Khandwala NS, Hyde RA, Besirli CG. Toxoplasma retinochoroiditis with chorioretinal neovascularization in a young patient. Case Rep Ophthalmol. 2021;12:259-263. doi:10.1159/000512286

13. Lin CJ, Chen SN, Hwang JF, Hu PS. Successful treatment of toxoplasmosis-associated choroidal neovascular lesions with bevacizumab and antiparasitic therapy. Retin Cases Brief Rep. 2011;5:348-351. doi:10.1097/ICB.0b013e3181ff09ae

14. Benevento JD, Jager RD, Noble AG, et al.; Toxoplasmosis Study G. Toxoplasmosis-associated neovascular lesions treated successfully with ranibizumab and antiparasitic therapy. Arch Ophthalmol. 2008;126:1152-1156. doi:10.1001/archopht.126.8.1152

15. Smith JR, Franc DT, Carter NS, Zamora D, Planck SR, Rosenbaum JT. Susceptibility of retinal vascular endothelium to infection with Toxoplasma gondii tachyzoites. Invest Ophthalmol Vis Sci. 2004;45:1157-1161. doi:10.1167/iovs.03-1105

16. Aggio FB, Novelli FJ, Rosa EL, Nobrega MJ. Combined branch retinal vein and artery occlusion in toxoplasmosis. Arq Bras Oftalmol. 2016;79:189-191. doi:10.5935/0004-2749.20160054

Kumar V, Takkar B, Chandra P, Kumar A. Macular infarction in a patient with Toxoplasma retinochoroditis. BMJ Case Rep. 2016;2016: bcr2015212596.

18. Azar G, Favard C, Salah S, Brezin A, Vasseur V, Mauget-Faysse M. Optical coherence tomography angiography analysis of retinal and choroidal vascular networks during acute, relapsing, and quiescent stages of macular toxoplasma retinochoroiditis. Biomed Res Int. 2020;2020:4903735. doi:10.1155/2020/4903735

19. de Oliveira Dias JR, Campelo C, Novais EA, et al. New findings useful for clinical practice using swept-source optical coherence tomography angiography in the follow-up of active ocular toxoplasmosis. Int $J$ Retina Vitreous. 2020;6:30. doi:10.1186/ s40942-020-00231-2

20. Vezzola D, Allegrini D, Borgia A, et al. Swept-source optical coherence tomography and optical coherence tomography angiography in acquired toxoplasmic chorioretinitis: a case report. J Med Case Rep. 2018;12:358. doi:10.1186/s13256-018-1902-x

21. Simsek M, Ozdal PC, Kocer AM. Optic nerve involvement in ocular toxoplasmosis: 12 year data from a tertiary referral center in Turkey. Arq Bras Oftalmol. 2019;82:302-309. doi:10.5935/0004-2749.20 190058

published pages including figures, diagrams and references. The manuscript management system is completely online and includes a very quick and fair peer-review system, which is all easy to use. Visit http://www.dovepress.com/testimonials.php to read real quotes from published authors. 